\title{
Sciendo
}

Int. J. of Applied Mechanics and Engineering, 2020, vol.25, No.2, pp.75-87

DOI: 10.2478/ijame-2020-0021

\section{USING THE ERFI FUNCTION IN THE PROBLEM OF THE SHAPE OPTIMIZATION OF THE COMPRESSED ROD}

\author{
J. MARCINOWSKI* and M. SADOWSKI \\ Chair of Building Constructions, Institute of Civil Engineering \\ University of Zielona Góra \\ Szafrana 1, 65 - 516 Zielona Góra, POLAND \\ E-mails: j.marcinowski@ib.uz.zgora.pl; miroslaw.sadowski@gmail.com
}

\begin{abstract}
The shape of the optimal rod determined in the work meets the condition of mass conservation in relation to the reference rod. At the same time, this rod shows a significant increase in resistance to axial force. In the examples presented, this increase was $80 \%$ and $117 \%$, respectively, for rods with slenderness of 125 and 175 . A practical benefit from the use of compression rods of the proposed shapes is clearly visible.

The example presented in this publication shows how great the utility in the structural mechanics can be, resulting from the applications of complex analysis (complex numbers). This approach to many problems can find its solutions, while they are lacking in the real numbers domains. What is more, although these are operations on complex numbers, these solutions have often their real representations, as the numerical example shows.

There are too few applications of complex numbers in the technique and science, therefore it is obvious that the use of complex analysis should have an increasing range.

One of the first people to use complex numbers was Girolamo Cardano. Cardano, using complex numbers, was solving cubic equations, unsolvable to his times - as the famous Franciscan and professor of mathematics Luca Pacioli put it in his paper Summa de arithmetica, geometria, proportioni et proportionalita (1494). It is worth mentioning that history has given Cardano priority in the use of complex numbers, but most probably they were discovered by another professor of mathematics - Scipione del Ferro (cf. [1]).

We can see, that already then, they were definitely important (complex numbers).
\end{abstract}

Key words: compressed rod, hollow rod, shape optimization, buckling criterion, analytical approach, Erfi function, program Mathematica ${ }^{\mathrm{TM}}$.

\section{Introduction}

Buckling is a special case of a wider group of phenomena referred to as loss of structural stability. Buckling is a sudden transition from one form of deformation: axial compression to a qualitatively different form of deformation - to bending. This phenomenon causes a rapid redistribution of internal forces, which is dangerous for the construction. Theoretically, if the compressed rod is perfectly symmetrical and its compressive force is perfectly axial and centric, there is exactly one deformation state, in which the balance is maintained. If, on the other hand, the influence of bending on the change of internal forces, the so-called second order effects, is taken into account, then an other solution is possible, which exists only when the compressive force reaches a certain value called the critical force.

For centuries, although this branch of science developed relatively recently, scientists have sought for such shapes of compressed elements, with which it was possible to reduce their mass. Thus, the search process leading to the minimization of the weight of the structure, while maintaining stability criteria, especially in spatial shaping, can be considered as an optimization of stability. In the ancient times, technical solutions, which used a slight bulge, thickening of the column's shaft in the Dorian style, by overlapping the expanding stone segments up to $2 / 3$ of the height - i.e. enthasis, were applied (see Fig.1). This procedure was designed to abolish the optical illusion, manifested in the impression of the concavity of the columns, as well as - presumably to increase the load capacity (comp. [2], [3]).

\footnotetext{
* To whom correspondence should be addressed
} 


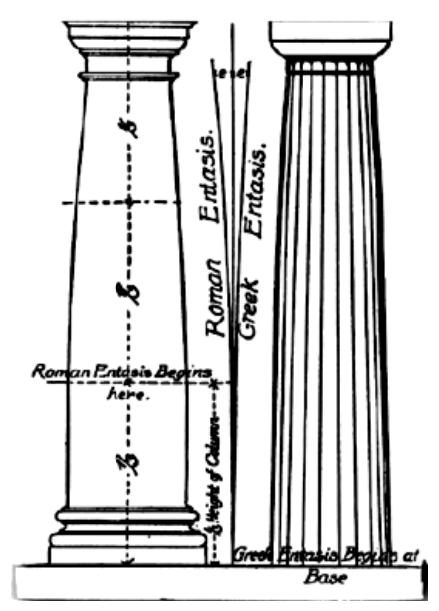

Fig.1. Enthasis.

The technical solutions referred to above, namely, structural elements with variable cross-sections along the axis (such elements are called non-prismatic rods or columns), are also located in the first and subsequent aircraft structures, such as spacers in aircrafts (biplanes), i.e. intentionally profiled rods, connecting lobes and hardening them at the same time They take the shape of non-prismatic poles, most commonly of circular cross-section, capable of transferring the desired force and, at the same time, their mass is as small as possible. In Fig. 2 we can see the example - type the FVM Ö1 Tummelisa aircraft, designed by Swedish army aircraft workshop.

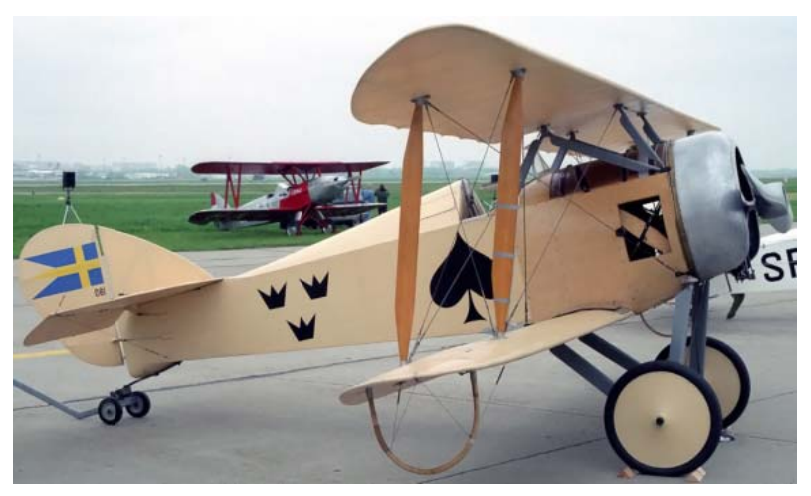

Fig.2. Replica of FVM Ö1 Tummelisa.

Other applications of structure stability optimization are presented in the paper by Krzyś (cf. [4]), where the author analyzes creating shape of bending beams, while Gajewski and Życzkowski (cf. [5]) provide a solution to the problem of optimal compression rod.

In conclusion, the problem of minimizing the weight of the structure is in fact the problem of the arrangement of the substance (metal, alloy or plastic, etc.) in the design, so that the construction element, after a conscious and, more importantly, deliberate redistribution of material, transfers the greatest possible force compression (cf. [6]). So it is a problem of optimizing the topology of the structure.

\section{Physical and mathematical model of the rod}

The present study analyzes a non-prismatic hollow rod with an annular cross-section, the solid of which is formed as follows: the forming outer and inner surfaces are the result of the rotation of accepted curves around the geometrical axis of rod. The curves in question are continuous flat curves. 
Let $r_{p}$ be the length of the external radius that forms the cross-section at the ends of the rod, $r_{m}-$ the length of the outer radius forming the cross-section at half the length of the rod, $L$ - the length of the rod, while $t$ - the thickness of the wall at half the length of the rod. We assume that the wall thickness of the rod is variable along its axis, where at its ends it is expressed by $\alpha t$, where $\alpha$ means the ratio of the wall thickness of the rod at its ends to the wall thickness of the rod at half its length (Fig.3).

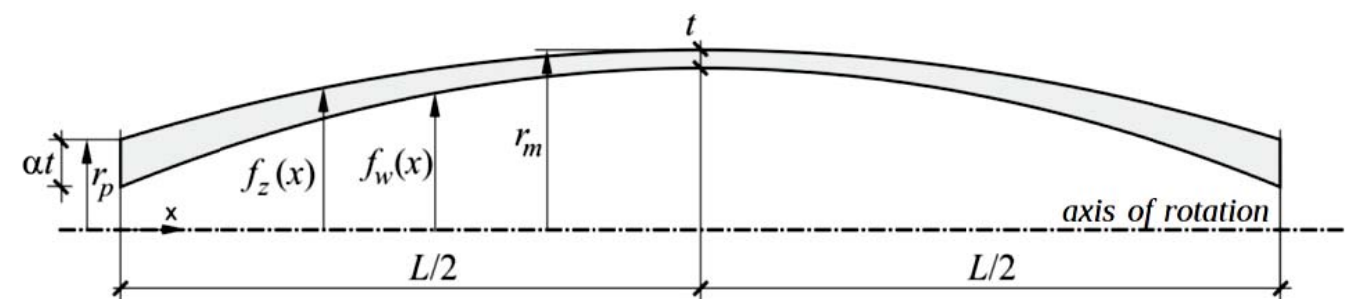

Fig.3. Cross section of the non-prismatic rod with a transverse ring section and variable wall thickness (scale not maintained).

The external and internal of the rotational surfaces are the result of the rotation of the bell curve graphs (other solutions can be found in [7]), given by the relation

$$
\begin{aligned}
& f_{z}(x)=\frac{r_{p}-r_{m}}{e^{1 / 4}-1} \exp \left(\frac{x}{L}-\frac{1}{2}\right)^{2}+\frac{e^{1 / 4} r_{m}-r_{p}}{e^{1 / 4}-1} \\
& f_{w}(x)=\frac{t-\alpha t+r_{p}-r_{m}}{e^{1 / 4}-1} \exp \left(\frac{x}{L}-\frac{1}{2}\right)^{2}+\frac{\left(\alpha-e^{1 / 4}\right) t+e^{1 / 4} r_{m}-r_{p}}{e^{1 / 4}-1}
\end{aligned}
$$

where $f_{z}(x)$ means a functional relation whose graphic image is the external forming solids of the rod, while $f_{w}(x)$ - the relation whose graphic image is the internal forming solids of the rod while $e$ denotes Euler's number known also as Neper's number (from the definition: $e=\lim _{n \rightarrow \infty} e=\lim _{n \rightarrow \infty}(1+1 / n)^{n} \approx 2.718 \ldots$ ) This number is of the base for natural logarithm.

Let us make an initial optimization assumption: the considered non-prismatic ring rod has the same mass as the mass of a standard cylindrical rod with the same length $L$; the radius length $r_{0}$ of its cross-section (Fig.4).

a)

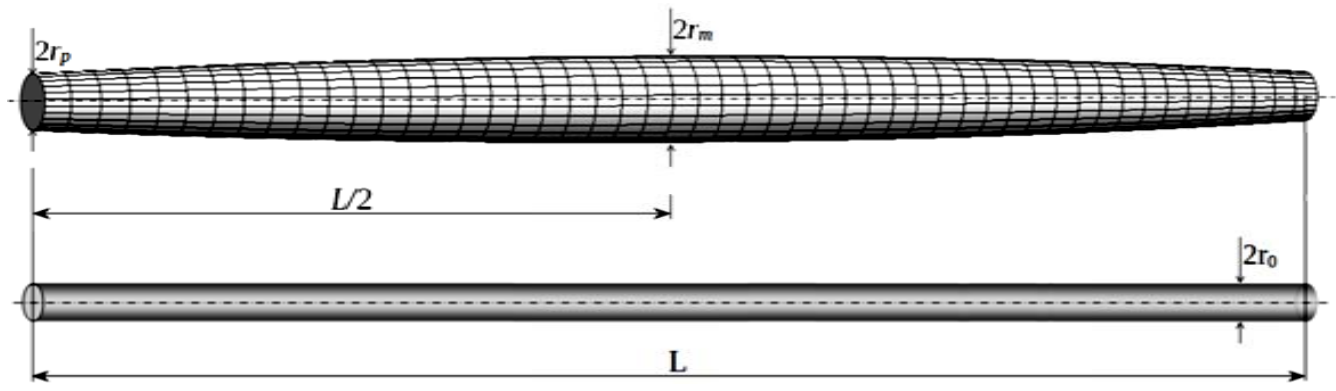

Fig.4. Considered rods: non-prismatic rod with transverse ring cross-section $(a)$, reference cylindrical rod $(b)$.

The above assumptions lead to the relation 


$$
\pi r_{0}^{2} L=\pi \int_{0}^{L}\left(f_{z}^{2}(x)-f_{w}^{2}(x)\right) d x
$$

which after some rearrangements (made in Mathematica ${ }^{\mathrm{TM}}$; comp. [8]) binds the radii of particular crosssections - at the ends of the rod and inside it

$$
r_{m}=r_{m}\left(r_{p}\right)=A / B
$$

where

$$
\begin{aligned}
& A=2\left(e^{1 / 4}-1\right)^{2} r_{0}^{2}+\left[2 e^{1 / 2}+4 e^{1 / 4}\left(-\sqrt{\pi} \operatorname{Erfi} \frac{1}{2}+\alpha\left(1-\sqrt{\pi} \operatorname{Erfi} \frac{1}{2}\right)\right)+\right. \\
& +\sqrt{2 \pi} \operatorname{Erfi} \frac{1}{\sqrt{2}}+2 \sqrt{\pi} \alpha\left(2 \operatorname{Erfi} \frac{1}{2}-\sqrt{2} \operatorname{Erfi} \frac{1}{\sqrt{2}}\right)+ \\
& \left.+\alpha^{2}\left(2-4 \sqrt{\pi} \operatorname{Erfi} \frac{1}{2}+\sqrt{2 \pi} \operatorname{Erfi} \frac{1}{\sqrt{2}}\right)\right] t^{2}+ \\
& -2\left[2 e^{1 / 4}\left(-1+\sqrt{\pi} \operatorname{Erfi} \frac{1}{2}\right)+\sqrt{\pi}\left(2 \operatorname{Erfi} \frac{1}{2}-\sqrt{2} \operatorname{Erfi} \frac{1}{\sqrt{2}}\right)+\right. \\
& \left.+\alpha\left(2-4 \sqrt{\pi} \operatorname{Erfi} \frac{1}{2}+\sqrt{2 \pi} \operatorname{Erfi} \frac{1}{\sqrt{2}}\right)\right] r_{p} t
\end{aligned}
$$

and

wherein

$$
\begin{aligned}
& B=2\left[2 e^{1 / 2}+2 e^{1 / 4}\left(-2 \sqrt{\pi} \operatorname{Erfi} \frac{1}{2}+\alpha\left(-1+\sqrt{\pi} \operatorname{Erfi} \frac{1}{2}\right)\right)+\right. \\
& \left.+\sqrt{\pi}\left(2 \alpha \operatorname{Erfi} \frac{1}{2}+\sqrt{2} \operatorname{Erfi} \frac{1}{\sqrt{2}}-\sqrt{2} \alpha \operatorname{Erfi} \frac{1}{\sqrt{2}}\right)\right] t
\end{aligned}
$$

$$
\operatorname{Erfi}(z)=-i \operatorname{erf}(i z)=-\frac{2 i}{\sqrt{\pi}} \int_{0}^{i z} \exp \left(-u^{2}\right) d u
$$

is a complex or imaginary error function (while the function $\operatorname{erf}(x)=\frac{2}{\sqrt{\pi}} \int_{0}^{x} \exp \left(-u^{2}\right) d u$, is referred to as the Gaussian error curve).

It follows from the definition (2.6) that

$$
\operatorname{Erfi}(1 / 2)=0.61495 \quad \text { oraz } \quad \operatorname{Erfi}(1 / \sqrt{2})=0.95344
$$

Let the rod under consideration be subjected to axial compression. We assume that it is simply supported at the ends and that it is burdened with initial geometrical imperfection, expressed as

$$
f(x)=e_{0} \sin \frac{\pi}{L} x
$$

where $e_{0}$ presents the amplitude $\left(e_{0}=L / 250[\mathrm{~mm}]\right)$. 
The graphic presentation of the above assumptions is shown in the static scheme (Fig.5).

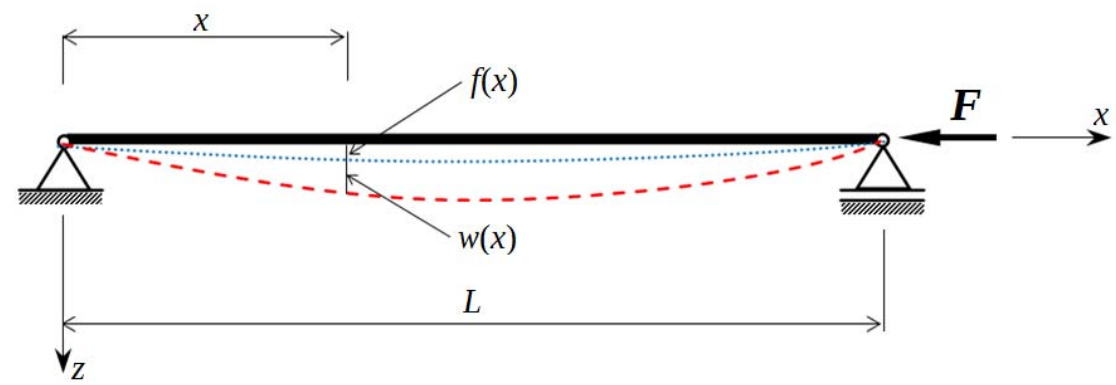

Fig.5. Static scheme of the system.

\section{The objective function and a set of constraints}

Due to the problem posed, the objective function is the maximum compressive force

$$
F=\max .
$$

Suppose that the rod is to work in the linearly elastic range. Therefore, the necessity of an examination of the problem arises: searching for the maximum compressive force, the value of which will not result in plasticizing the material of the $\operatorname{rod}\left(F_{u p l}\right)$

$$
F_{u p l}=\max \cdot F_{u p l(\max )}=F \text {. }
$$

In the case of the yield force, the relationship should be maximized

$$
F_{u p l}=f_{y} \cdot A\left(\frac{L}{2}\right) \cdot \frac{I\left(\frac{L}{2}\right)}{I\left(\frac{L}{2}\right)+\frac{L}{250} \cdot r_{m} \cdot A\left(\frac{L}{2}\right)}
$$

wherein

- $f_{y}$ - yield stress of the steel used to make the rod,

- $A(x)$ - variable cross-section area $\left[\mathrm{mm}^{2}\right]$ is defined by the relationship

$$
A(x)=\pi\left(f_{z}^{2}(x)-f_{w}^{2}(x)\right)
$$

- $I(x)$ - variable moment of inertia of the cross-section $\left[\mathrm{mm}^{4}\right]$ is given by the relationship

$$
I(x)=\frac{\pi}{4}\left(f_{z}^{4}(x)-f_{w}^{4}(x)\right)
$$

Relation (3.3) follows from the relations (3.6) expressing maximal stresses at the middle section of the rod (comp. Fig.6) and its limitation by the yield stress $f_{y}$ 


$$
\sigma_{\max }=\frac{F}{A\left(\frac{L}{2}\right)}+\frac{F \cdot e_{0} \cdot r_{m}}{I\left(\frac{L}{2}\right)} \leq f_{y} .
$$

It was assumed that theimperfection amplitude $e_{0}$ is equal to $L / 250$ and $F=F_{u p l}$ and the inequality was replaced by the equality. After relatively simple transformations the formula (3.3) was obtained.

The same formula can be obtained on the basis of formula 6.44 from Eurocode EN 1993-1-1 (2005): Eurocode 3: Design of steel structures - Part 1-1: General rules and rules for buildings, if one direction bending is assumed.

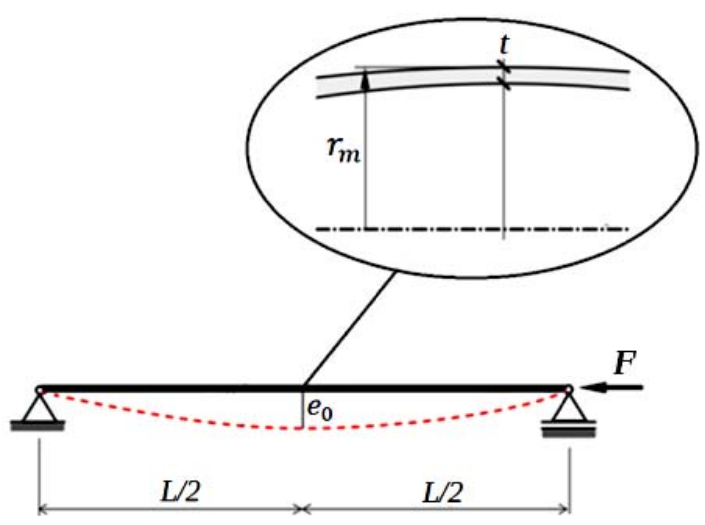

Fig.6. Geometry of the rod at its middle section.

Let us move to the optimization of the rod's shape. It will be based on the search for the extreme of force (3.3) for two independent decision variables:

a) wall thickness at half the length of the $\operatorname{rod}(t)$,

b) the ratio of the wall thickness at the ends of the rod to the wall thickness in the middle $(\alpha)$.

Remaining geometrical parameters $r_{p}$ and $r_{m}$ are defined by formulae (2.3) to (2.5), against the assumption that $r_{p}=L / 25$ (comp. (3.9)).

Let us define a set of constraints.

- Geometric constraints:

- equality of rod masses (this condition has already been used in relations (2.3), (2.4) and (2.5))

$$
m_{p(r)}=m_{p(d)}
$$

where $m_{p(r)}$ - mass of the reference rod, $m_{p(d)}$ - mass of the searched, hollow rod.

- positive wall thickness value at half the length of the rod

$$
t>0
$$

- the value of the external radius that forms the rod's solid at its end is constant and equal

$$
r_{p}=L / 25,
$$

- positive value of the wall thickness ratio at the ends of the rod to the wall thickness in its half 


$$
\alpha>0 .
$$

- Strength constraints:

- impassability of the yield stress $\left(f_{y}\right)$ at the end of the rod and half its length

$$
\sigma_{r z}(0) \leq f_{y}, \quad \sigma_{r z}(L / 2) \leq f_{y}
$$

- impassability of the critical stress values of the cylindrical shell (local loss of stability of the compressed cylindrical shell) at the end of the rod and half its length

$$
\sigma_{r z}(0) \leq \sigma_{g r(p)}(0), \quad \sigma_{r z}(L / 2) \leq \sigma_{g r(s)}(L / 2)
$$

where the symbol $\sigma_{r z}$ denotes the actual stresses in the bar (or, more precisely, in the wall), while additionally the limit stresses are described by the relations

$$
\begin{aligned}
& \sigma_{g r(p)}(0)=\frac{1}{\sqrt{3\left(1-v^{2}\right)}} \frac{E \alpha t}{R(0)}, \\
& \sigma_{g r(s)}(L / 2)=\frac{1}{\sqrt{3\left(1-v^{2}\right)}} \frac{E t}{R(L / 2)},
\end{aligned}
$$

wherein

$$
R(x)=\frac{1}{2}\left(f_{z}(x)+f_{w}(x)\right)
$$

The search for the extreme (the maximum, to be more precise) of the force (3.3), was carried out in the Mathematica ${ }^{\mathrm{TM}}$ program. This process is fully implemented by the Maximize procedure (making it possible to find the global maximum of functions in the area defined by the limitations), although in the given problem, due to the complexity of calculations, a "derivative command" was used, using an iterative (numerical) sequence - N Maximize, performing the same task. It was requested that the iterative pattern would use the stochastic optimization method, namely the Differential Evolution algorithm (the differential evolution results from Ken Price's attempts to solve the problem of Chebyshev polynomials suggested by Rainer Storn, when Ken Price invented a way to distort the vector population using vector differences. The algorithm was officially published in 1997 in the paper: Price, K. and Storn R. "Differential Evolution." Dr Dobb's J., Issue 264, 18-24 and 78, Apr. 1997.). The method available in the referenced program ensures that complicated calculations based on non-linear relations are carried out, and what is more - it is "a simple, yet effective evolutionary algorithm, solving the problem of global continuous optimization" (cf. [9]). The number of iterations was limited to 500 .

At this point, it is worth remembering about another optimization problem, more fundamental, namely, it can be assumed that the shape of a cylindrical tube should be determined, which will also show the feature of transferring the increased value of compressive force. However, as shown by the calculations, the shape of the rod proposed in the article definitely is more beneficial than the shape of the cylindrical tube in the considered context. 


\section{Numerical examples}

Let us look at two numerical examples. Let the geometrical and physical parameters of the cylindrical reference rod be as follows:

- case 1:

- cross-section radius: $r_{0}=4 \mathrm{~mm}$,

- rod length: $\quad L=250 \mathrm{~mm}$,

- rod slenderness: $\quad \lambda=L / i=2 L / r_{0}=125$,

- material - steel: $\quad E=2.110^{5} \mathrm{MPa}, v=0.3, f_{y}=235 \mathrm{MPa}$,

- case 2:

- cross-section radius: $\quad r_{0}=10 \mathrm{~mm}$,

- rod length: $\quad L=875 \mathrm{~mm}$,

- rod slenderness: $\quad \lambda=L / i=2 L / r_{0}=175$,

- material - steel: $\quad E=2.1 \cdot 10^{5} \mathrm{MPa}, v=0.3, f_{y}=235 \mathrm{MPa}$.

The results of the analysis performed (optimization) are presented in Tab.1. Figures 6 and 7 present axial cross-sections of the determined shapes of rods (In both figures, the cross-section of the reference rod is marked with a dotted line.)

Table 1. A summary of the values of decision variables and critical forces of reference, tubular and optimal rods.

\begin{tabular}{|c|c|c|c|c|c|c|c|c|c|c|}
\hline \multirow{4}{*}{$\begin{array}{c}\text { Length } \\
L \\
{[\mathrm{~mm}]}\end{array}$} & \multirow{4}{*}{$\begin{array}{c}\text { Slend } \\
\text { erness } \\
\lambda\end{array}$} & \multirow{4}{*}{$\begin{array}{c}\text { Yield } \\
\text { stress } \\
f_{y} \\
{[\mathrm{MPa}]}\end{array}$} & \multirow{4}{*}{$\begin{array}{c}\begin{array}{c}\text { Reference } \\
\text { rod }\end{array} \\
\text { value of } \\
\text { max. } \\
\text { compressive } \\
\text { strength } \\
F_{\text {ref }(\max )} \\
{[N]}\end{array}$} & \multirow{4}{*}{$\begin{array}{c}\begin{array}{c}\text { Tubular } \\
\text { rod }\end{array} \\
\text { value of max. } \\
\text { compressive } \\
\text { strength } \\
F_{\text {rur }}(\max ) \\
{[N]}\end{array}$} & \multicolumn{6}{|c|}{ Optimal rod } \\
\hline & & & & & \multirow{3}{*}{\begin{tabular}{|c} 
value of max. \\
compressive \\
strength \\
$F_{\text {opt }(\max )}$ \\
{$[N]$}
\end{tabular}} & \multirow{3}{*}{$\begin{array}{c}\text { increase in } \\
\text { load } \\
\text { capacity in } \\
\text { relation to } \\
\text { the ref. rod } \\
\quad[\%]\end{array}$} & \multicolumn{4}{|c|}{ decision variable values } \\
\hline & & & & & & & \multirow[t]{2}{*}{$\alpha$} & \multicolumn{3}{|c|}{$[\mathrm{mm}]$} \\
\hline & & & & & & & & $t$ & $r_{p}$ & $r_{m}$ \\
\hline 250 & 125 & 235 & 5906.2 & 7096.7 & 10624.4 & 80 & 1.47 & 0.50 & 10.0 & 16.3 \\
\hline 875 & 175 & 275 & 35997.4 & 55802.5 & 78121.4 & 117 & 1.24 & 1.05 & 35.0 & 49.9 \\
\hline
\end{tabular}

\section{Characteristics and application of Erfi functions in the issue under consideration}

As a result of the initial assumption, namely due to assuming the equality of the masses of the considered non-prismatic ring rod and the standard cylindrical rod of the same length, we obtained a relationship that expresses the relation between certain geometric parameters of the non-prismatic rod between the outer radius of the solid in the middle of its length and the outer radius of the solid of the rod at its ends (cf. relations (2.2) - (2.4)). We can see that in the relations referred to, there is the special Erfi function, which was defined by the relation (2.5). It is worth emphasizing that in a general form, it is a complex function of a complex variable. 


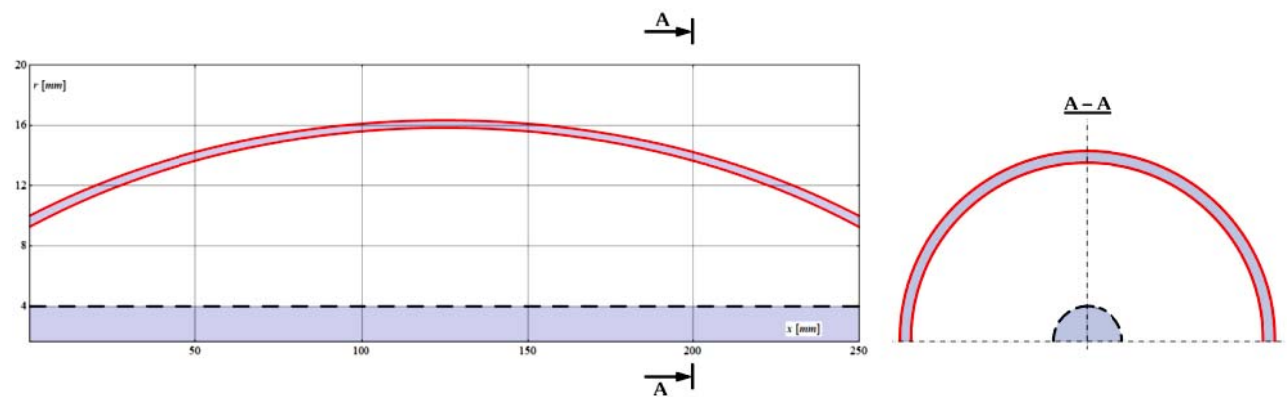

Fig.7. Axial cross-section of the upper half of the optimal $\operatorname{rod}(L=250 \mathrm{~mm}, \lambda=125)$ dashed line - crosssection of the upper half of the reference bar (scale not maintained).

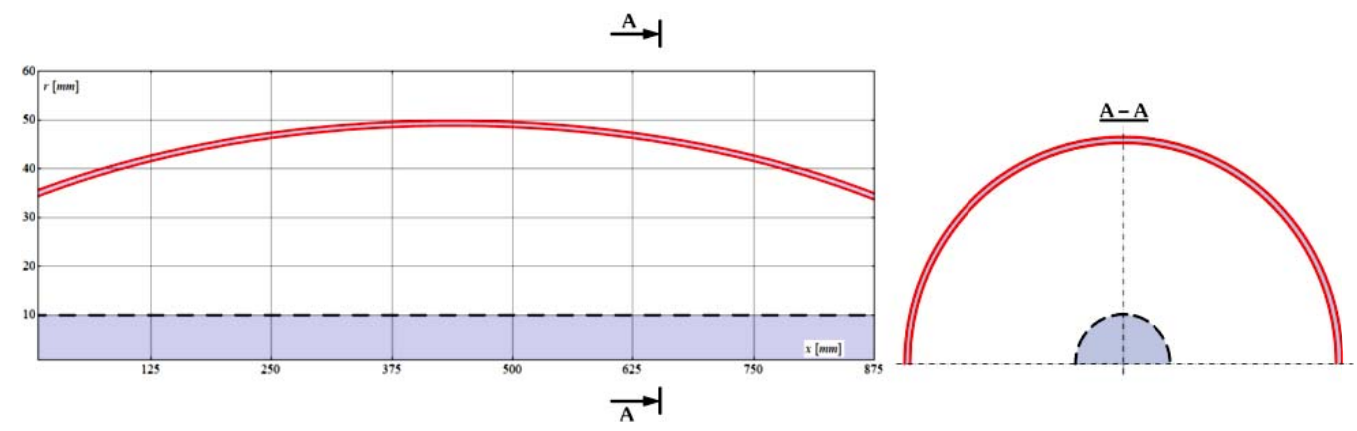

Fig.8. Axial cross-section of the upper half of the optimal $\operatorname{rod}(L=875 \mathrm{~mm}, \lambda=175)$ dashed line - crosssection of the upper half of the reference rod (scale not maintained).

There is no need to convince anyone of the benefits of using complex numbers and their analysis. They find application e.g. in quantum mechanics, where the wave function, which is a solution to the general Schrödinger equation, represents the location of particles in the configuration space and time is a function with complex values. In addition, complex numbers are used in electrical engineering, in which, thanks to algebralization of differential-integral equations by mapping current and voltage waveforms in the form of a symbolic function, a simplified analysis of alternating current circuits is enabled (cf. [10]).

And although in some important issues of structural mechanics there are elements, among others, in the vibration theory, or in the search for solutions for certain differential equations describing various issues(meaning the Laplace transform and Fourier transform.) or the complex Żukowski function known in aerodynamic (cf. [11]), which transforms the flow around the circular profile into the flow around the Żukowski profile, used to profile the aircraft wings, compared to other technical areas, the use of complex numbers is too low in technique. It is a challenge that is still being attempted: to develop the use of complex analysis in structural mechanics. This work is, in a way, just such an attempt.

Let us take a closer look at the function that is the subject of the present discussion. The special Erfi function is a complex function of a complex variable. It has a number of interesting properties that can be found in the literature of the subject. These are the selected ones

$$
\begin{aligned}
& \frac{d}{d z} \operatorname{Erfi}(z)=\frac{2}{\sqrt{\pi}} \exp \left(z^{2}\right), \\
& \int \operatorname{Erfi}(z) d z=z \operatorname{Erfi}(z)-\frac{1}{\sqrt{\pi}} \exp \left(z^{2}\right),
\end{aligned}
$$




$$
\begin{aligned}
& \operatorname{Erfi}(z)=\frac{2}{\sqrt{\pi}} \sum_{k=0}^{\infty} \frac{z^{2 k+1}}{k !(2 k+1)} \\
& \operatorname{Erfi}(z)=-i+\frac{1}{\sqrt{\pi}} \exp \left(z^{2}\right)\left(z^{-1}+\frac{1}{2} z^{-3}+\frac{3}{4} z^{-5}+\frac{15}{8} z^{-7}+\ldots\right) .
\end{aligned}
$$

The last relation can be transformed by introducing

$$
z=x+i y
$$

Therefore

$$
\begin{aligned}
& \operatorname{Erfi}(z)=\operatorname{Erfi}(x, y)=-i+\frac{1}{\sqrt{\pi}} \exp (x+i y)^{2}\left((x+i y)^{-1}+\frac{1}{2}(x+i y)^{-3}+\right. \\
& \left.+\frac{3}{4}(x+i y)^{-5}+\frac{15}{8}(x+i y)^{-7}+\ldots\right)= \\
& =-i+\frac{1}{\sqrt{\pi}} \exp \left(x^{2}-y^{2}\right)(\cos \cos (2 x y)+i \sin (2 x y))\left[\left(\frac{x}{x^{2}+y^{2}}+\frac{1}{2} \frac{x^{3}-3 x y^{2}}{\left(x^{2}+y^{2}\right)^{3}}+\right.\right. \\
& \left.+\frac{3}{4} \frac{x^{5}-10 x^{3} y^{2}+5 x y^{4}}{\left(x^{2}+y^{2}\right)^{5}}+\frac{15}{8} \frac{x^{7}-21 x^{5} y^{2}+35 x^{3} y^{4}-7 x y^{6}}{\left(x^{2}+y^{2}\right)^{7}}\right)+ \\
& \left.i\left(\frac{-y}{x^{2}+y^{2}}+\frac{1}{2} \frac{-3 x^{2} y+y^{3}}{\left(x^{2}+y^{2}\right)^{3}}+\frac{3}{4} \frac{-5 x^{4} y+10 x^{2} y^{3}-y^{5}}{\left(x^{2}+y^{2}\right)^{5}}++\frac{15}{8} \frac{-7 x^{6} y+35 x^{4} y^{3}-21 x^{2} y^{5}+y^{7}}{\left(x^{2}+y^{2}\right)^{7}}\right)+\ldots\right]
\end{aligned}
$$

The above shows that

$$
\begin{aligned}
& \operatorname{Re}[\operatorname{Erfi}(z)]=\frac{1}{\sqrt{\pi}} \exp \left(x^{2}-y^{2}\right)\left[\operatorname { c o s } \operatorname { c o s } ( 2 x y ) \left(\frac{x}{x^{2}+y^{2}}+\frac{1}{2} \frac{x^{3}-3 x y^{2}}{\left(x^{2}+y^{2}\right)^{3}}+\right.\right. \\
& \left.+\frac{3}{4} \frac{x^{5}-10 x^{3} y^{2}+5 x y^{4}}{\left(x^{2}+y^{2}\right)^{5}}+\frac{15}{8} \frac{x^{7}-21 x^{5} y^{2}+35 x^{3} y^{4}-7 x y^{6}}{\left(x^{2}+y^{2}\right)^{7}}+\ldots\right]+ \\
& -\sin (2 x y)\left(-1+\frac{-y}{x^{2}+y^{2}}+\frac{1}{2} \frac{-3 x^{2} y+y^{3}}{\left(x^{2}+y^{2}\right)^{3}}+\frac{3}{4} \frac{-5 x^{4} y+10 x^{2} y^{3}-y^{5}}{\left(x^{2}+y^{2}\right)^{5}}+\right. \\
& \left.+\frac{15}{8} \frac{-7 x^{6} y+35 x^{4} y^{3}-21 x^{2} y^{5}+y^{7}}{\left(x^{2}+y^{2}\right)^{7}}+\ldots\right)
\end{aligned}
$$

and 


$$
\begin{aligned}
& \operatorname{Im}[\operatorname{Erfi}(z)]=\frac{1}{\sqrt{\pi}} \exp \left(x^{2}-y^{2}\right)\left[\operatorname { s i n } ( 2 x y ) \left(\frac{x}{x^{2}+y^{2}}+\frac{1}{2} \frac{x^{3}-3 x y^{2}}{\left(x^{2}+y^{2}\right)^{3}}+\right.\right. \\
& \left.+\frac{3}{4} \frac{x^{5}-10 x^{3} y^{2}+5 x y^{4}}{\left(x^{2}+y^{2}\right)^{5}}+\frac{15}{8} \frac{x^{7}-21 x^{5} y^{2}+35 x^{3} y^{4}-7 x y^{6}}{\left(x^{2}+y^{2}\right)^{7}}+\ldots\right]+ \\
& +\cos (2 x y)\left(-1+\frac{-y}{x^{2}+y^{2}}+\frac{1}{2} \frac{-3 x^{2} y+y^{3}}{\left(x^{2}+y^{2}\right)^{3}}+\frac{3}{4} \frac{-5 x^{4} y+10 x^{2} y^{3}-y^{5}}{\left(x^{2}+y^{2}\right)^{5}}+\right. \\
& \left.+\frac{15}{8} \frac{-7 x^{6} y+35 x^{4} y^{3}-21 x^{2} y^{5}+y^{7}}{\left(x^{2}+y^{2}\right)^{7}}+\ldots\right)
\end{aligned}
$$

and

$$
\operatorname{Abs}[\operatorname{Erfi}(x, y)]=|\operatorname{Erfi}(x, y)|=\sqrt{\operatorname{Re}^{2}[\operatorname{Erfi}(x, y)]+\operatorname{Im}^{2}[\operatorname{Erfi}(x, y)]} .
$$

The graphs of the real part (25), the imaginary part (26) and the module (27) of the Erfi function, as well as their contour plots, are presented Fig.9.

a)
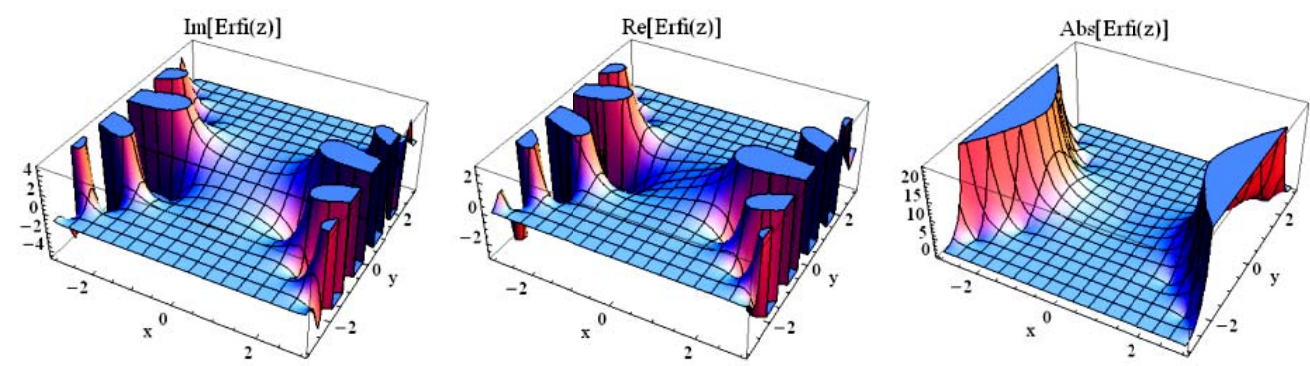

b)
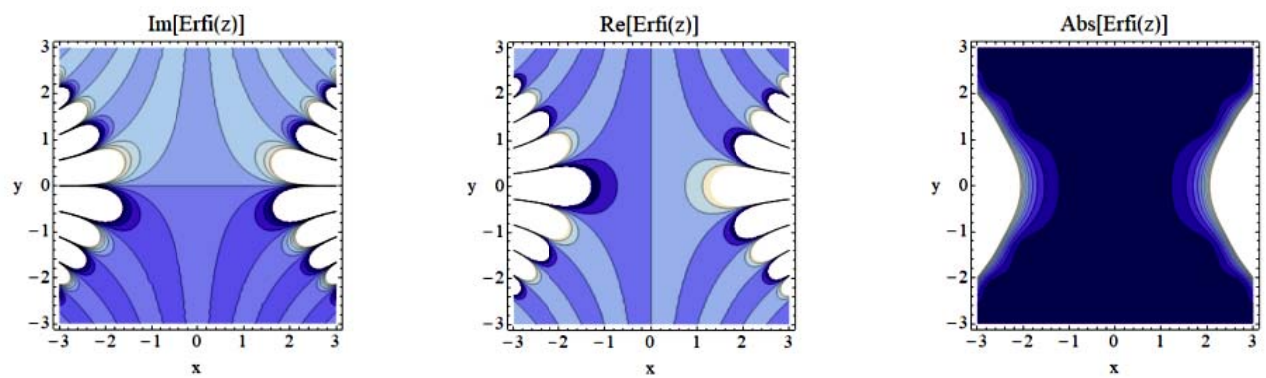

Fig.9. Graph of the imaginary part, the real part and the Erfi function module $(z)$ for $z \in \mathbb{C}(a)$ and contour plots $(b)$.

However, the most important thing from the point of view of mechanics is the observation that the function being examined becomes a real function, when its domain is narrowed down to the set of real numbers, on which the analysis of mechanics problems is usually based. It turns out that this function can be presented in the form 


$$
\operatorname{Erfi}(x)=\frac{2}{\sqrt{\pi}} \int_{0}^{x} \exp \left(u^{2}\right) d u
$$

which renders it possible to interpret it as a field under the curve $f(x)=\frac{2}{\sqrt{\pi}} \exp \left(x^{2}\right)$ (within the integral).

a)

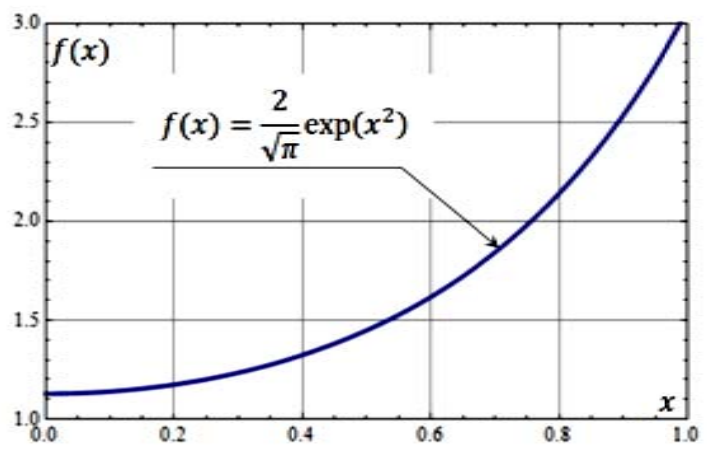

b)

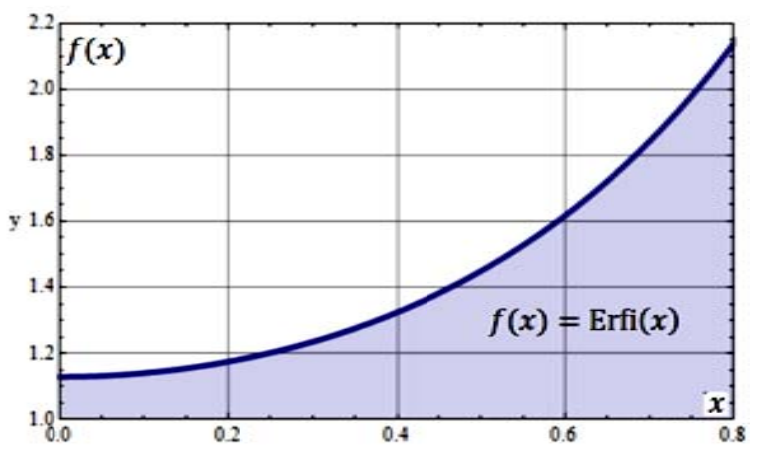

Fig.10. Graph $f(x)=\frac{2}{\sqrt{\pi}} \exp \left(x^{2}\right)(a)$ and geometric interpretation of the Erfi function for the real variable $(b)$.

The form of the function (5.6) suggests that it is a continuous and differentiable function (which is easy to demonstrate). Therefore, we can rightly assume that the relationship defining the size of $r_{m}$ represents a valuable relationship, from a technical point of view.

\section{Nomenclature}

$e-$ Euler's number known also as Neper's number

$e_{0}-$ amplitude of bow imperfection

$F_{\text {ref(max) }}{ }^{-}$value of max. compressive strength (reference rod)

$\left.F_{\text {rur (max) }}\right)^{-}$value of max. compressive strength (tabular rod)

$F_{\text {opt }(\max )}-$ value of max. compressive strength (optimal rod)

$f_{y}-$ yield stress

$L$ - length of the rod

$r_{m}-$ length of the outer radius forming the cross-section at half the length of the rod

$r_{p}-$ length of the external radius that forms the cross-section at the ends of the rod

$t-$ thickness of the wall at half the length of the rod

$\alpha-$ ratio of the wall thickness of the rod at its ends to the wall thickness of the rod at half its length

$\lambda$ - slenderness

\section{References}

[1] Miś B. (2008): Secret e number and other secrets of mathematics.- Scientific and Technical Publishing House, Warsaw.

[2] Thompson P., Papadopoulou G. and Vassiliou E. (2007):The origins of entasis: illusion, aesthetics or engineering?-Spatial Vision, vol.20, No.6, pp.531-543. 
[3] Marcinowski J. and Sadowski M. (2016): Buckling capacity of non-prismatic rods with polygonal cross-sections.In: Sustainable Construction, the University Publishing House of the University of Technology and Life Sciences in Bydgoszcz, Bydgoszcz.

[4] Krzyś W. (1968): Optimale formen gedrückter dünnwandiger stützen in elastisch-plastischen bereich. - Wiss. Z. Tech., Univ. Dresden, vol.17, No.2, pp.407-410.

[5] Gajewski A. and Życzkowski M. (1998): Optimal structural design under stability constraints.- Kluwer Academic Publishers, Dordrecht, Boston, London.

[6] Bochenek B. and Krużelecki J. (2007): Optimization of construction stability. Contemporary problems.- Cracow University of Technology Publisher, Cracow.

[7] Marcinowski J. and Sadowski M. (2015): Shape optimization of non-prismatic rods of circular hollow crosssections and of variable wall thickness.-In: Proceedings of the stability of structures: XV-th symposium. Zakopane, Poland, 2018. Łódź: Department of Strength of Materials and Structures of the Lodz University of Technology, pp.99-100.

[8] Gliński H., Grzymkowski R., Kapusta A. and Słota D. (2012): Mathematica 8. - Publishing House of the Jacek Skalmierski Computer Laboratory, Gliwice.

[9] Opara K. (2014): Analysis of the differential evolution algorithm and its application in the determination of statistical dependencies.- Abstract of the Doctoral Thesis.

[10] Bobrowski C. (1995): Physics - Short Course.- Warsaw: Scientific and Technical Publishing House.

[11] Stasiak J. and Walden H. (1971): Mechanics of liquids and gases in sanitary engineering. - Arkady Publishing House, Warsaw.

[12] Rykaluk K. (2012): Problems of stability of metal structures. - Lower Silesian Educational Publishing House, Wrocław.

Received: January 25, 2020

Revised: April 6, 2020 